\title{
New Design and Architecture of a Smart Tracker: Flexible and Scalable for PV and CSP Systems
}

\author{
Kamal Anoune ${ }^{1,2}$, Mohsine Bouya ${ }^{1}$ and Abdellatif Ben Abdellah ${ }^{1,2}$ \\ 1. Renewable Energy and Advanced Materials Laboratory, International University of Rabat, Parc Technopolis, Sala Al Jadida \\ 11100, Morocco \\ 2. Innovation and Management of Industrial Systmes Laboratory, College of Science and Techniques of Tanger, University \\ Abdelmalek Essaidi, Tanger 90 100, Morocco
}

Received: November 19, 2014 / Accepted: December 12, 2014 / Published: March 31, 2015.

\begin{abstract}
This work proposes a new design and architecture of a flexible biaxial solar tracker. A new approach was adopted with the use of a two separated cards, the smart and power card in a scalable concept. This module allows a more saving of energy in comparison with the fixed systems for PV (photovoltaic) application and allows hire performances for CSP (concentrated solar power) systems. It provides a significant added value for higher power applications in comparison with the existing system. The developed sun tracking system is autonomous, flexible, scalable and low cost system.
\end{abstract}

Key words: Smart sun tracker, flexible architecture, PV systems, CSP systems, energy saving.

\section{Introduction}

The consumption of electricity keeps only increase, and prices of fossil fuels follow the same trends: Where does the interest to find alternatives such as renewable energy? Which is an ecological and an inexhaustible source? Several studies are made to improve the energy efficiency of its systems, especially photovoltaic systems that are experiencing large global expansion. Nevertheless a numerical study [1] shows that the use of a biaxial solar tracker allows the annual increase energy efficiency by $36 \%$ relative to a fixed PV (photovoltaic) system, a comparison of energy performance [2] shows that, we can collect more than $31 \%$ energy by using a biaxial solar tracker over a fixed $\mathrm{PV}$ installation.

The variation in the energy efficiency of photovoltaic systems using a biaxial solar tracker resides in the mechanical structure and the electronic design of the solar tracker itself, which intervenes in

Corresponding author: Mohsine Bouya, associate professor, research fields: renewable energies and microelectronics. E-mail: mohsine.bouya@uir.ac.ma. the importance of our new design of biaxial solar tracker, there are architectural design [3] similar to our solar tracker, involving microcontroller architecture, but for surveillance application, other design involving PLC (programmable logic controller) for realization of the design of the solar tracker [4] in vocation to maximize energy efficiency.

The position of the sun changes constantly, both during the day but also during different periods of the year. The objective of this work is to design and develop an intelligent solar tracker, modular and bi-axial "Smart Tracking System" to guide solar systems at best compared to the positioning of the sun in order to increase productivity. "Smart Tracking System" is able to collect the maximum light intensity from the sun. This system was designed as flexible and adaptable for several applications, as a tracker of the position of the sun, such as solar radiation is perpendicular to the flat surface of the PV panels for electrical application and focused on the collector of CSP (concentrating solar power) systems for thermal application, which can produce the maximum power 
electrical gain and the efficient thermal performances respectively, with very less power consumption. The system consists of two modular cards: a control card and a power one. The first one allows the processing of the signal from the external sensor that is designed based on a network of LDR (light dependent resistor) $[3,5,6]$, and then runs an algorithm servo position and generates the control signals for the power board, which allows a better control of the motors driving the mechanical structure to the azimuthal and vertical movements of the using systems (PV or CSP). "Smart Tracking System" has an LCD (liquid crystal display) two-line alphanumeric display for displaying system status: search mode or night mode, ambient weather parameters in real time as the internal and external temperature, the level of light intensity, the wind speed and the offset value. This system also has an internal operating temperature control fan, and protection against excessive wind speed. All the features of the system including the execution of the servo, regulations and protection of the display management are controlled by a microcontroller algorithm [7].

\section{Functional Diagram}

Fig. 1 presents the global functional diagram which describes the functioning of the solar tracker, it includes a section dedicated to the measurement of physical values in real time such as wind speed, internal and external temperature sensor (CSP application), the luminosity of the LDR network and also logical values such as the state of the limit detectors. The second part focuses on the description of the power card that has the distinction of being flexible and adaptable, it controls indeed azimuthal movement and elevation movement, generated following the instructions from the control card. The third part looks at the description of the functionality of the control card, its role is to monitor and control the system of solar tracker, and indeed, it ensures priority the regulation of the sun's position in real time to the using information collected from its Network-LDR sensor. It also controls the ambient parameters, either the indoor and outdoor temperature, the state of LDR, wind speed, the state of the thermostat, and the state of limit switches and displays its information through its LCD display, using a scrolling menu incorporated in its 18F4550 PIC (programmable integrated circuit) microcontroller.

\section{Electronic Control Card}

The circuit (Fig. 2) shows an electronic design of the system with different modules, it is designed using the ISIS Proteus 7.10 software tool [8], the module includes several modules around the 18F4550 PIC microcontroller, the left part consists of a system for measuring the light intensity (networks of four LDR), with the information about the limit of safety part and the output display. The middle part comprises a LM35 sensor for measuring the ambient temperature, next microcontroller which manages and controls the entire electronic system.

The right part includes an alphanumeric display with contrast adjustment potentiometer, the circuit possesses a button to reset the digital system, and two buttons for scrolling display pages, it also has a command to output power the small fan, and finally three independent analog inputs.

This design (Fig. 2) is used to test the functionality of the electronic system, and also allows for the measurement of current and voltage of the various electronic components, it indeed facilitates electronic design, based on the actual functions that circuit must ensure and through the adaptation of input/output signals and program management by the microcontroller.

\section{Description of System Modules}

The global view of the "Smart Tracking System" module is presented in Fig. 3.

\subsection{Electronic Control Card}

This circuit is equipped with the latest technology in the field of digital electronics based PIC 18F4550 microcontroller. As below described, this new design, providing great flexibility and high availability: 


\section{Wind Sensor Module:}

- Anemometer for measuring the wind speed;

- Adaptation of the electrical signal with the control card.
Internal temperature sensor module:

- Measures the ambient temperature of the card;

- Protection against the deterioration of the card.
Solar LDR sensor module:

- Detection of signals perpendicular to solar;

- Acquisition and adaptation of the electrical signal with the control card.

\section{DL \\ Programmable control Card: \\ - Flexible Programmable control card and Purely a lot of application; \\ - 8 Analog inputs, 1 input to relay, 1 relay output, 8 I/O OOC (on-off-control); \\ - Microcontroller PIC 18F4550: information processing; \\ - Running position control algorithm; \\ - System calibration and compensation LDRs voltage drop; \\ - Digital display 2 lines: management services display; \\ - Interface signals adaptation, communication with other modules; \\ - Bright LED (light emitting diode) and alarm signal.}

\section{TIL}

Power Card:

- Adaptable card power supply voltages Motor Very varied $(12 \mathrm{~V}=>30 \mathrm{VDC}$ (voltage direct current) $15 \mathrm{~A})(110 \mathrm{~V}=>240 \mathrm{VAC}$ (voltage alternating current) $10 \mathrm{~A})$

- Logic power electronics command motors positioning of the PV panel;

- Limit switch.

Drivers of azimuthal and elevation positioning:

(12 V $\Rightarrow 30$ VDC until $15 \mathrm{~A})$,

$(110 \mathrm{~V}=>240 \mathrm{VAC}$ until $10 \mathrm{~A})$

Limit switch:
Azimuthal position;
Elevation position.

Fig. 1 Functional diagram.

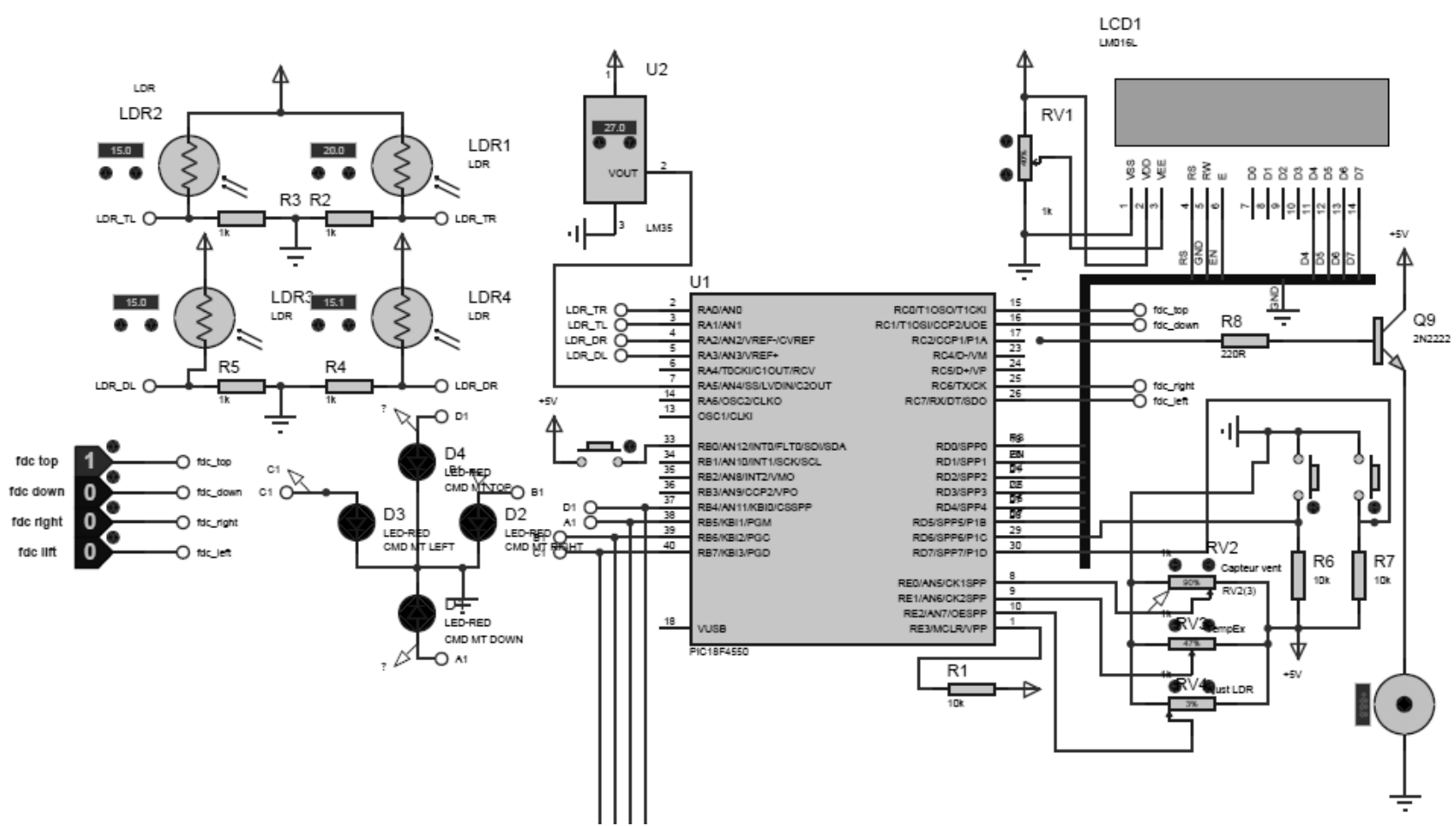

Fig. 2 Electronic layout of control card.

External temperature sensor module:

- Thermostat CSP (optional);

- Analog temp (optional). 


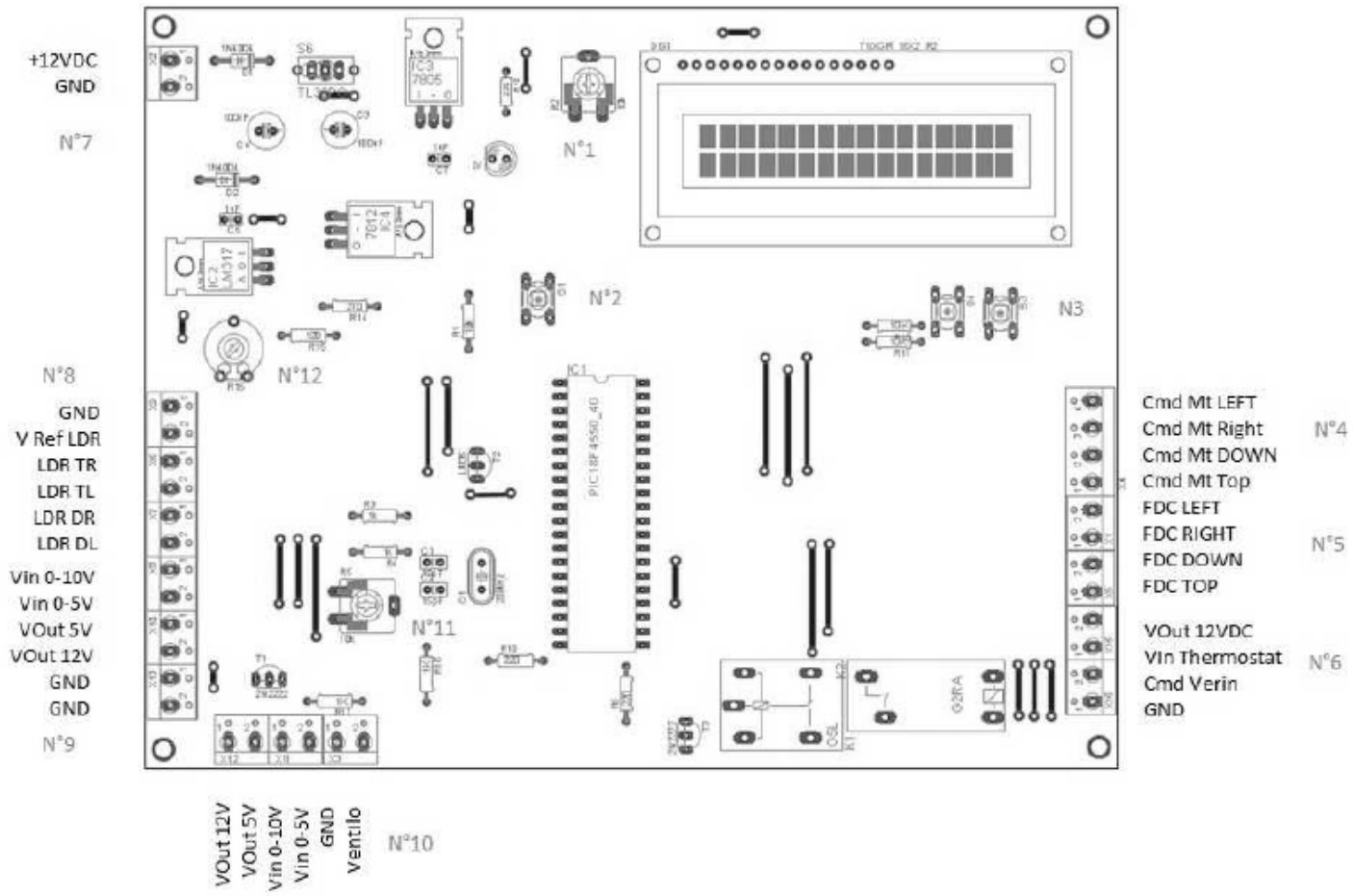

Fig. 3 Global view of the "Smart Tracking System" PCB (printed circuit board).

- A PIC microcontroller 18F4550, with a large computing filling through its arithmetic and logic unit ALU dedicated to the application of monitoring and control in real time, the program memory is relatively quite large:

- LCD 2-line alphanumeric display allows the consultation of the state of operation of the tracking and management of alarms and faults;

- Interfacing with other system modules that collect information and action on the position of the PV panel.

The modules are designed specifically for "Smart Tracking System", these modules is actually adapted to the control card:

- Alternative power action on the side of engine position tracking of the sun;

- The Systems for acquisition information about the positioning of the sun;

- Alternative power card and other modules voltage.

\subsection{Module of Repositioning Sensor}

This module uses the discipline of instrumentation and measurement, the sensor is composed of a network of four LDRs arranged in a cross geometry, separated with opaque walls and covered with a transparent plexi-glass, dedicated to the determination of the position of the sun by comparing the light intensity converted to the voltage of each LDR (Fig. 4).

It communicates, in real time, the available information of the sun to the control card. Principle scheme described as follow (Fig. 5).

Information must be received, conditioned and then transmitted to the control card, below a demonstrative diagram, it illustrates the signal processing, which characterized by different electronic floor:

- The LDRs are mounted in four-point network, and geometrically arranged in quadruple as possible as to locate the geometric sun position;

- Packaging \& signal adaptation according to the operating variables of the control card.

\subsection{Power Board for Motor Control}

The modular power card allows the tracking system to position the solar panel so that it is perpendicular to 


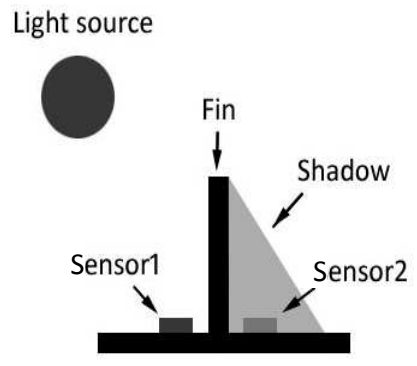

Fig. 4 Seen on the solar light capture principle using LDR.

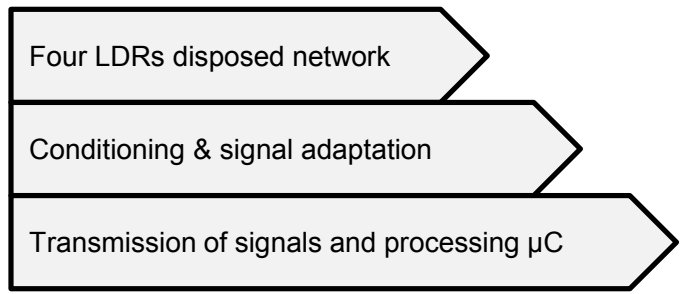

Fig. 5 Information processing flow.

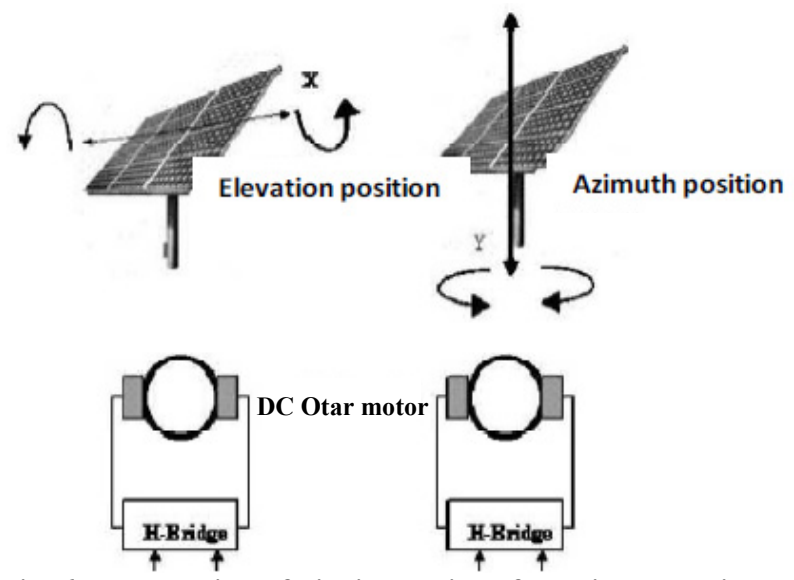

Fig. 6 Illustration of biaxial motions following the azimuth and elevation.

the solar radiation, which allows the solar panel to produce the maximum possible energy throughout the day. "Solar Tracking System" has two training systems driven by two servo engines (Fig. 6):

- an azimuthal positioning engine;

- an elevation positioning engine.

The modular electronic card (Fig. 7) "Flexible Power Card" has as function:

- manage both engines (elevation \& azimuth) for positioning the solar panel;

- provide energy (current and voltage) required to operate the engine;

- protection against short circuits and overload;

- contact the mother board;
- interfacing with limit switches.

\subsection{Wind Sensor Module}

This module is designed to measure the wind speed for safety reasons operation of PV panel. Indeed, if the wind speed exceeds a certain critical speeds, it may damage the supporting structure, this module includes:

- aerodynamic palette;

- dynamo-tachometer for measuring the wind speed;

- adaptation of the electrical input signal with the motherboard.

\subsection{Temperature Sensor Module}

To protect the PCB against deterioration caused by extreme conditions, "Solar Tracking System" is equipped with a temperature sensor (LM35) that measures in real time, the ambient temperature of the system and communicates information to the microcontroller which treats and displays it on the LCD screen, the system is also equipped with a regulatory function of the ambient temperature, which makes it possible to control a fan on exceeding the preprogrammed temperature.

\section{Results of Experience}

In this experiment, the results of measurements are analyzed during a sunny day in May, 2014, two support configurations for PV panels are used. The panels are identical in technology and power. A fixed configuration uses the panels in the south direction, with an elevation of $30^{\circ}$ and the other uses our "Smart tracking system".

We have taken a series of measures for the two systems (PV), fixed and mobile, the results are presented in Fig. 8.

By analyzing the instantaneous measurement results of the power of both PV systems (Fig. 8), we conclude that, the system with tracker presents the best energy optimization which reaches $40 \%$ relative to the fixed system. 


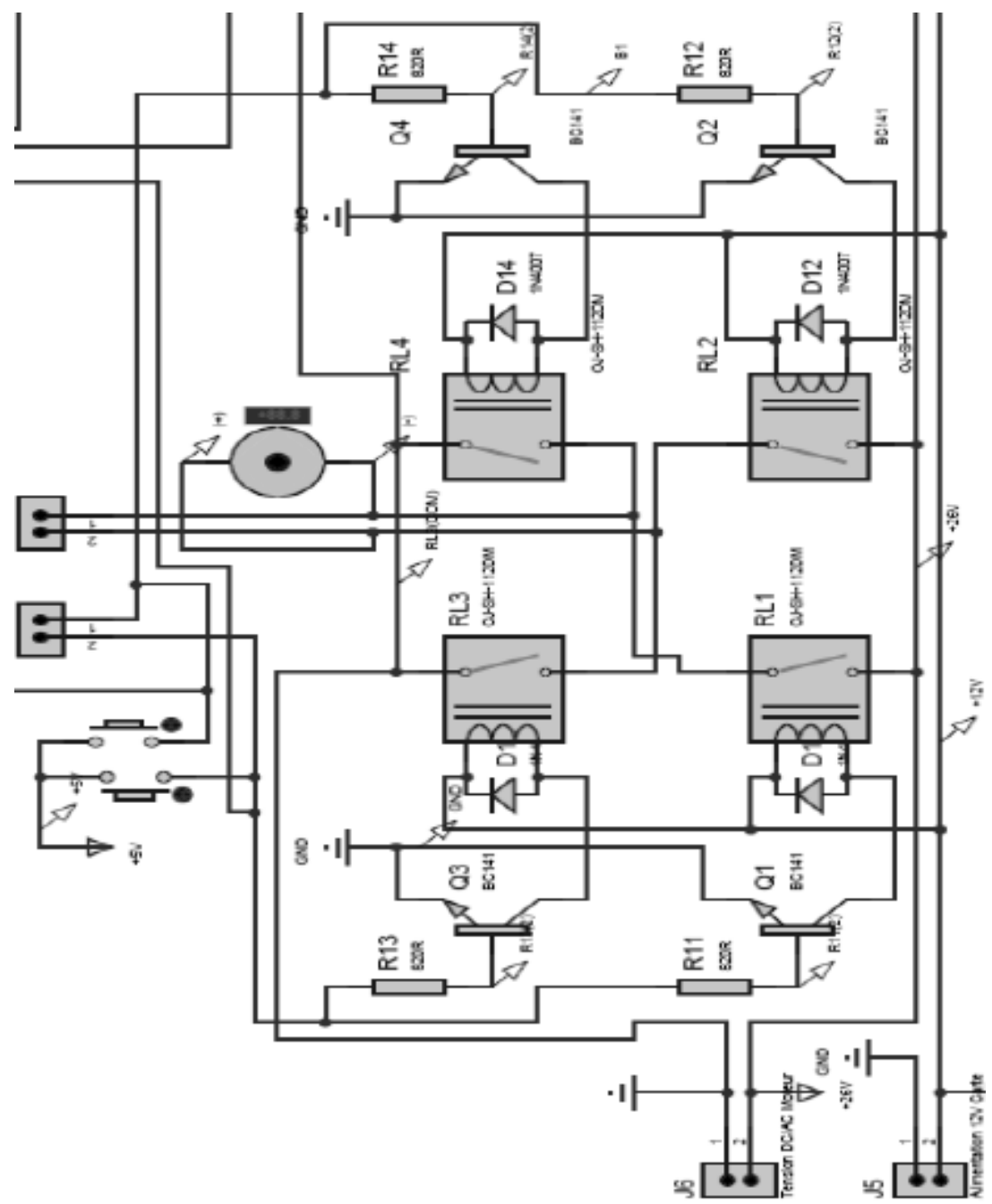

Fig. 7 Electronic layout of power card.

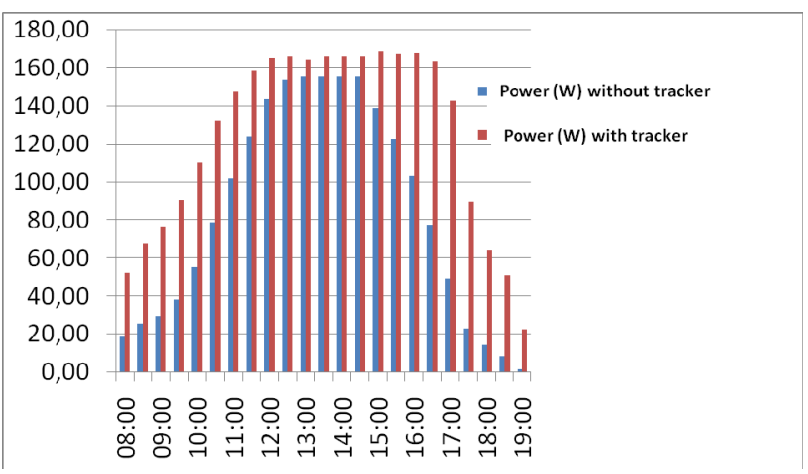

Fig. 8 Measurements of power collected from the PV with and without solar tracker.

\section{Conclusions}

The developed sun tracking system is adapted for PV and CSP renewable energy technologies and applications. It allows more energy efficiency and saving of these systems, with very less power consumption.

After design and realization of the smart control and power cards, a functional test is realized and demonstrates the added value of this system in comparison with the fixed panel for PV application. The experimental results demonstrate more than $40 \%$ increasing of energy efficiency with the developed tracking system. In the perspective, we investigate the experimental measurements for the CSP technology for heat water application, an adequate and new safety position will be investigated.

\section{Acknowledgments}

We would like to emphasize that, we have not been able to complete this research without the joint support 
of all employees of the renewable energy laboratory and its hierarchical superior of the UIR (International University of Rabat). We take this opportunity to express our deep gratitude and greeting to Elouahabi, M. for his inspiring and invaluable advice and availability. We express our special recognition and thanks to Ms. Najmeddine, F. Z. for her exemplary guidance, monitoring and constant encouragement.

\section{References}

[1] Zhang, P., Zhou, G., Zhu, Z., and Zhixiong, C. W. L. 2013. "Numerical Study on the Properties of an Active Sun Tracker for Solar Streetlight." Mechatronics 23 (September): 1215-22.

[2] Eke, E., and Senturk, A. 2012. "Performance Comparison of a Double-Axis Sun Tracking versus Fixed PV System." Solar Energy 86 (July): 2665-72.

[3] Soumen, G., and Haldar, N. 2014. "Solar Tracking System
Using AT89C51 Microcontroller and LDR.” International Journal of Emerging Technology and Advanced Engineering 4 (12): 403-7.

[4] Figueiredo, J. M. G., Sà da Costa, J. M. G., and Ramalho, R. R. 2008. "Intelligent Sun-Tracking System for Efficiency Maximization of Photovoltaic Energy Production.” Technical University Lisbon.

[5] Ponniran, A., Hashim, A., and Joret, A. 2011 "A Design of Low Power Single Axis Solar Tracking System Regardless of Motor Speed." International Journal of Integrated Engineering 3 (3): 5-9.

[6] Samuel, B. S., and Mrudula, J. 2013. "Design of Intelligent Solar Tracker Robot for Surveillance." International Journal of Advanced Research in Electrical, Electronics and Instrumentation Engineering 2 (10): 5147-51.

[7] Meeravali, S., and Suthagar, S. 2012. "Automatic Solar Tracker Robot." International Journal of Engineering Research \& Technology 1 (6): 2278-81.

[8] Kansal, R., and Singh, M. 2009 "PIC Based Automatic Solar Radiation Tracker." Asian Journal of Chemistry 21 (10): S091-094. 